\title{
Clinical assessment of deficits after SAH: hasty neurosurgeons and accurate neurologists
}

\author{
Christian Fung · Jürgen Beck $\cdot$ Lara Lauber • \\ René Müri · Andreas Raabe · Thomas Nyffeler
}

Received: 19 January 2012/Revised: 14 March 2012/Accepted: 15 March 2012/Published online: 14 April 2012

(C) Springer-Verlag 2012

\begin{abstract}
For survivors of aneurysmal subarachnoid hemorrhage (SAH), somatic and cognitive deficits can affect long-term outcomes. We were interested in comparing the deficits identified in SAH patients, including cognitive deficits, at discharge by neurosurgeons and deficits identified by neurologists upon admission to the rehabilitation unit on the same day. The assessment of deficits might have an impact on referring patients to rehabilitation. This retrospective study included $494 \mathrm{SAH}$ patients treated between 2005 and 2010. Of these, 50 patients were discharged to an affiliated rehabilitation unit. Deficits were grouped into 18 categories and summarized into three groups: major somatic, minor somatic, and cognitive deficits. Major somatic deficits were identified in 16 and 20 patients $(p=0.53)$, minor somatic deficits in 16 and $44(p<0.0001)$ patients, and cognitive deficits in 36 and $45(p<0.04)$ patients by neurosurgeons and neurologists, respectively. The absolute number of deficits in daily activities identified by the neurosurgeon and neurologist were 21 and 31 major somatic deficits $(p=0.2), 18$ and 97 minor somatic deficits $(p<0.0001)$, and 61 and 147 cognitive deficits $(p<0.0001)$, respectively. Significant differences in assessment of cognitive and minor somatic deficits between neurosurgeons and neurologists exist. Based on these findings, it is evident that for the neurosurgeon, there needs to be an increased awareness of the
\end{abstract}

C. Fung · J. Beck - L. Lauber - A. Raabe

Department of Neurosurgery, Bern University Hospital,

Freiburgstrasse 10, 3010 Bern, Switzerland

R. Müri · T. Nyffeler ( $₫)$

Department for Cognitive and Restorative Neurology,

Bern University Hospital, Freiburgstrasse 10, 3010 Bern,

Switzerland

e-mail: thomas.nyffeler@insel.ch assessment of cognitive deficits and a more routine interdisciplinary approach, including the use of neuropsychological evaluations, to ensure a better triage of patients to rehabilitation or for discharge home.

Keywords Aneurysmal subarachnoid hemorrhage . Assessment · Patient outcome - Cognitive deficit . Somatic deficit

\section{Introduction}

Aneurysmal subarachnoid hemorrhage (SAH) is a devastating disease with a high mortality and morbidity due to the initial bleeding and subsequent neurovascular events. Despite recent advances in surgical, anesthesiological, interventional procedures, and neurointensive care, the outcome for $\mathrm{SAH}$ patients remains poor [1]. Up to $80 \%$ of patients show a reduced health-related quality of life and deficits in neuropsychological functioning 1 year after SAH [2-5]. Cognitive impairment is one factor that greatly contributes to the reduction in quality of life for these patients $[2,6]$.

In the hospital setting, the treating physician is responsible for the assessment of cognitive deficits during acute therapy after SAH and any subsequent referral to a rehabilitation unit. For SAH patients, this role is frequently performed by the neurosurgeon. The pivotal question is whether neurosurgeons identify these deficits sufficiently and accurately-including cognitive deficits. To investigate this question, we reviewed the medical records of patients with somatic or cognitive deficits that were transferred to a rehabilitation unit. We compared the neurosurgeon's assessment of the patient at discharge to the rehabilitation unit to the assessment performed by the admitting neurologist at the rehabilitation unit on the same day. 


\section{Materials and methods}

We conducted a retrospective database study of $494 \mathrm{SAH}$ patients that were treated at the Department for Neurosurgery and the Institute for Cognitive and Restorative Neurology, Bern University Hospital, from January 2005 to December 2010. Of these, 50 consecutive SAH patients were discharged from the neurosurgery department and on the same day admitted to the rehabilitation unit at the Institute for Cognitive and Restorative Neurology. We collected the patient's clinical status by reviewing and recording all neurological deficits that were documented in the patient's medical record including assessments at discharge by the neurosurgeon, and admission to the rehabilitation unit by the neurologist. In total, 32 neurosurgical residents and 15 neurological residents performed the exams.

Deficits were grouped according to major somatic deficits (major cranial nerve deficit, major motor deficit, bedridden, parenteral or nasogastric tube feeding), minor somatic deficits (minor cranial nerve deficit, minor motor deficit, sensitivity, reflex status, supported mobility), and cognitive deficits (orientation, speech, apraxia, attention, executive function, memory, visuoconstruction, visual function, and neglect) (Table 1). The identified deficits were then summarized as the absolute number of deficits according to major somatic, minor somatic, and cognitive deficits, and as the number of patients having a documented deficit: major, minor, or somatic.

Patients who were unable to participate in a clinical examination due to a low level of vigilance or reduced compliance were not documented as having a deficit, rather they were documented as having an incomplete assessment for the particular parameter.

Descriptive statistics were calculated [mean and standard deviation (SD)] and we compared groups using a twosided Fisher exact $t$ test (GraphPad InStat, GraphPad Software Inc. La Jolla, CA, USA). Statistical significance was defined as $p<0.05$.

\section{Results}

Of the patients included in the study $(n=50), 29$ were female and 21 were male. The mean age was 52.5 years (SD 8). On admission to the neurosurgical department, two patients presented with a SAH Hunt/Hess grade I, 19 patients with grade II, ten patients with grade III, nine patients with grade IV, and ten patients with an SAH grade V. Upon discharge, a ventriculo-peritoneal shunt was placed in 22 patients due to malresorptive hydrocephalus. All but three patients were discharged from the neurosurgical unit and admitted to the rehabilitation clinic on the
Table 1 Overview of deficit categories

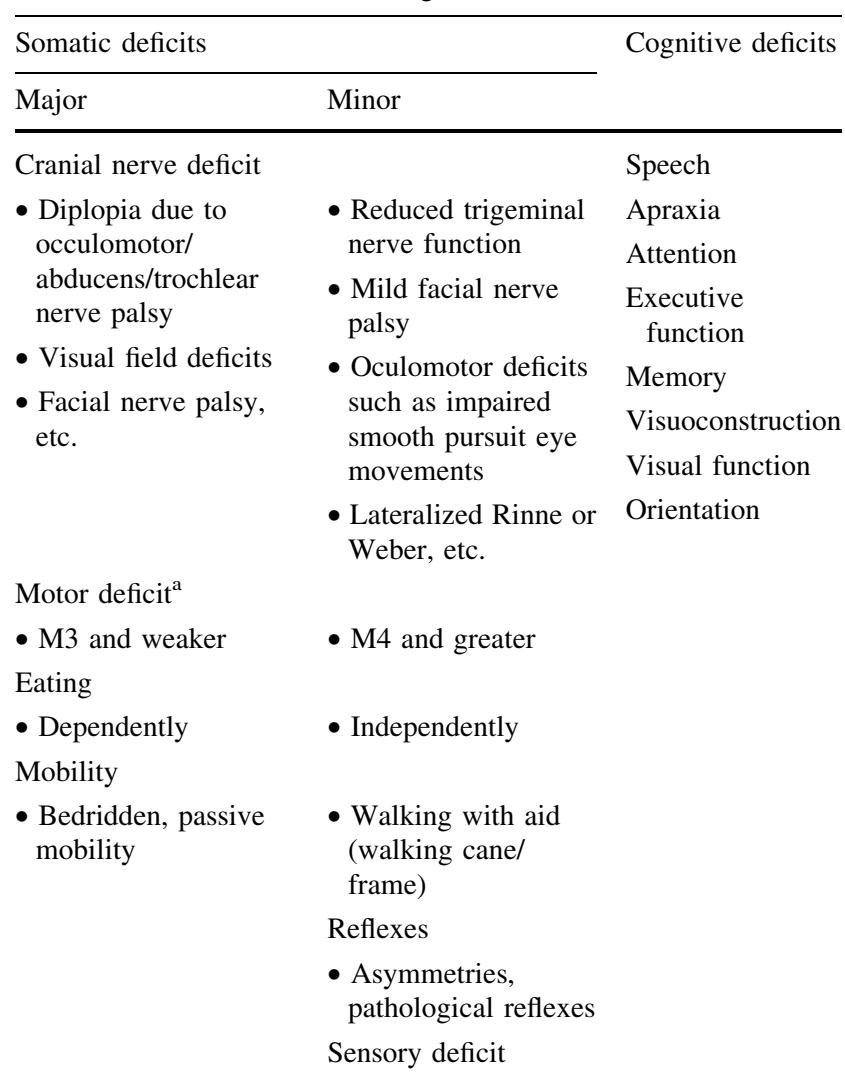
${ }^{a}$ Muscle strength was assessed according to the British Medical
Research Council Scale

same day. The three remaining patients were transferred to a third hospital and then readmitted to the rehabilitation clinic 1 week $(n=1)$ and 2 weeks $(n=2)$ after discharge from the neurosurgical unit.

When evaluating overall deficits, there were 100 deficits identified in the neurosurgeon's discharge assessment compared to 275 deficits at the neurologist's admission assessment (Fig. 1a; Table 2). Further analyses by deficit category showed that neurosurgery discharge listed 21 and neurological admission assessment listed 31 major somatic deficits $(p=0.2), 18$ and 97 minor somatic deficits $(p<$ $0.0001)$, and 61 and 147 cognitive deficits $(p<0.0001)$, respectively.

In the assessment of the number of patients identified with a deficit, neurosurgeons and neurologists documented major somatic deficits in 16 and 20 patients $(p=0.53)$, minor somatic deficits in 16 and 44 patients $(p<0.0001)$, and cognitive deficits in 36 and 45 patients $(p<0.04)$, respectively (Fig. 1b; Table 2).

For both disciplines, cognitive deficits exceeded major somatic deficits in all but one Hunt and Hess grade. In Hunt and Hess grade 1, neurosurgeons assessed one major somatic deficit without a cognitive deficit. Incomplete assessment was noted for 11 parameters in neurosurgical 

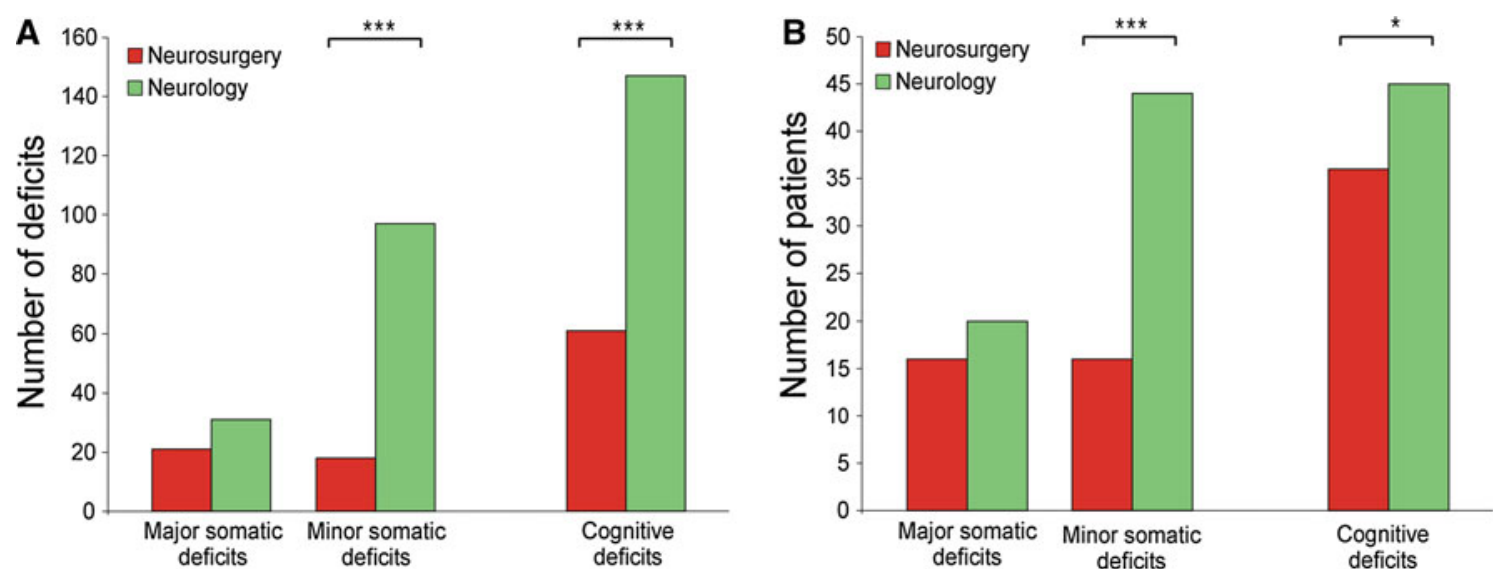

Fig. 1 Absolute number of deficits per group and specialty (a) and number of patients with a documented deficit per group between specialties (b)

Table 2 Overall results per specialty: absolute numbers of deficits recorded and number of total patients with documented deficit

\begin{tabular}{lcll}
\hline & Major somatic & Minor somatic & Cognitive \\
\hline Absolute number of deficits & & \\
Neurosurgery & 21 & 18 & 61 \\
Neurology & 31 & 97 & 147 \\
Number of patients with deficits $(n=50)$ & \\
Neurosurgery, $(n, \%)$ & $16(32)$ & $16(32)$ & $36(72)$ \\
Neurology, $(n, \%)$ & $20(40)$ & $44(88)$ & $45(90)$ \\
\hline
\end{tabular}

(two major somatic, two minor somatic deficits, seven cognitive deficits) and 85 times in neurological assessments (six major somatic, two minor somatic deficit, 77 cognitive deficits). The number of incomplete cognitive assessments varied significantly between the two disciplines $(p<0.0001)$.

\section{Discussion}

We found that considerable differences in clinical assessment exist between neurosurgeons and neurologists, even though the assessments were made on the same day and in a highly select patient group-those already selected for rehabilitation. We observed the widest gap for minor neurological deficits that might have less impact on daily life. However, the most relevant difference exists in the assessment of cognitive deficits. These deficits were significantly less often diagnosed by neurosurgeons, whereas neurologists identified significantly more patients with cognitive deficits. Taking into consideration that an incomplete assessment for a cognitive parameter was noted 77 times by neurologists and only seven times by neurosurgeons, this might even accentuate our results. According to the neurological assessment, 45 out of 50 patients do show a cognitive deficit. This finding is further supported by recent publications that report a high incidence of cognitive deficits and the impact on daily life in $\mathrm{SAH}$ patients [2, 3, 5, 7].

We suspect that the significant differences between the two specialties in recognizing cognitive and minor somatic deficits may be explained by the time spent on assessment and perspective with respect to the rehabilitation consequences of the deficits. Furthermore, our findings highlight a difference in medical training, in terms of the focus on cognitive and somatic deficit assessments, between the two specialties. This not only resulted in the difference of assessed deficits, but also may be responsible for a more differentiated assessment especially of cognitive deficits. The reduced awareness for neurosurgeons to identify cognitive deficits might also be caused by using rather robust outcome scales after SAH. The most often used outcome scales in SAH are the modified Rankin Scale (mRS) and Glasgow Outcome Scale (GOS). Neither of these scales directly include cognitive deficits [8]. This might influence the assessment behavior of neurosurgeons shifting the focus away from cognitive deficits. It is only recently that papers have been published with more sophisticated outcome parameters including health-related quality of life, cognitive impairment, and emotional problems $[2,6,7]$. This reflects the growing interest and the meaning of cognitive impairment after SAH.

We are aware that training and function varies considerably between the two specialties and, generally speaking, neurosurgeons do not conduct cognitive assessments in their daily routine. However, because treatment of SAH patients does not end after acute care, for the neurosurgeon, an increased awareness of cognitive deficits is required and an interdisciplinary approach, including neuropsychologists, should be utilized. 
The weaknesses of the study include its retrospective nature, the small number of patients, and single center design. Despite these drawbacks, this is the first study that highlights the differences between the neurosurgical and neurological assessments in SAH. Further research is needed to corroborate the findings.

Cognitive deficits are underestimated in neurosurgical practice. This might impact referral patterns to rehabilitation, especially in patients with good outcome using the $\mathrm{mRS}$ and GOS. The detection of cognitive deficits and referral to specialized therapy has the potential to provide benefit to a large group of patients [9]. This indicates the need for a neuropsychological assessment to identify cognitive deficits in acute care for a better triage of patients to rehabilitation or for discharge home [7]. We conclude that neurosurgeons should be more aware of the frequency of cognitive deficits after SAH.

Acknowledgments No funding was received for this work. Dr. Thomas Nyffeler had full access to all the data in the study and had final responsibility for the decision to submit for publication. The authors received editorial support for the final version of the manuscript by Susan Wieting, Bern University Hospital, Department of Neurosurgery, Publications Office, Bern, Switzerland.

Conflicts of interest None.

\section{References}

1. Molyneux AJ, Kerr RS, Yu LM et al (2005) International Subarachnoid Aneurysm Trial (ISAT) of neurosurgical clipping versus endovascular coiling in 2143 patients with ruptured intracranial aneurysms: a randomised comparison of effects on survival, dependency, seizures, rebleeding, subgroups, and aneurysm occlusion. Lancet 366:809-817

2. Meyer B, Ringel F, Winter Y et al (2010) Health-related quality of life in patients with subarachnoid haemorrhage. Cerebrovasc Dis 30:423-431

3. Hackett ML, Anderson CS (2000) Health outcomes 1 year after subarachnoid hemorrhage: an international population-based study. The Australian Cooperative Research on Subarachnoid Hemorrhage Study Group. Neurology 55:658-662

4. Visser-Meily JM, Rhebergen ML, Rinkel GJ, van Zandvoort MJ, Post MW (2009) Long-term health-related quality of life after aneurysmal subarachnoid hemorrhage: relationship with psychological symptoms and personality characteristics. Stroke 40:15261529

5. Powell J, Kitchen N, Heslin J, Greenwood R (2002) Psychosocial outcomes at three and nine months after good neurological recovery from aneurysmal subarachnoid haemorrhage: predictors and prognosis. J Neurol Neurosurg Psychiatry 72:772-781

6. Springer MV, Schmidt JM, Wartenberg KE, Frontera JA, Badjatia N, Mayer SA (2009) Predictors of global cognitive impairment 1 year after subarachnoid hemorrhage. Neurosurgery 65:10431050

7. Passier PE, Visser-Meily JM, van Zandvoort MJ, Post MW, Rinkel GJ, van Heugten C (2010) Prevalence and determinants of cognitive complaints after aneurysmal subarachnoid hemorrhage. Cerebrovasc Dis 29:557-563

8. Kim DH, Haney CL, Van Ginhoven G (2005) Utility of outcome measures after treatment for intracranial aneurysms: a prospective trial involving 520 patients. Stroke 36:792-796

9. Saciri BM, Kos N (2002) Aneurysmal subarachnoid haemorrhage: outcomes of early rehabilitation after surgical repair of ruptured intracranial aneurysms. J Neurol Neurosurg Psychiatry 72:334337 